\title{
A hybrid approach of modular Planning - synchronizing factory and building planning by using component based synthesis
}

\author{
Jan Winkels \\ TU Dortmund \\ University \\ Jan.winkels@tu \\ -dortmund.de
}

\author{
Julian Graefenstein \\ TU Dortmund \\ University \\ Julian.graefenstein@tu- \\ dortmund.de
}

\author{
Lisa Lenz \\ TU Dortmund \\ University \\ Lisa.lenz@tu- \\ dortmund.de
}

\author{
Kai Weist \\ TU Dortmund \\ University \\ Kai.weist@tu- \\ dortmund.de
}

\author{
Kevin Krebil \\ TU Dortmund \\ University \\ Kevin.krebil@tu- \\ dortmund.de
}

\author{
Mike Gralla \\ TU Dortmund \\ University \\ Mike.gralla@tu- \\ dortmund.de
}

\begin{abstract}
The more and more rising complexity of the industrial environment is triggering companies in a way that is more challenging than ever before. Not only are factory planning projects difficult to handle because of the dynamics and complexity also the necessary planning of the accompanied building gets more and more difficult. To handle this complexity and reduce time and effort for planning as a major factor of success the mainly separately done planning aspects needs to be synchronized. This paper will show an approach of a hybrid factory-building planning method in order to be able to shorten planning time and effort. By using a constraint solving technique the necessary planning tasks are aligned partly automatically and will be processed as a useful planning workflow in form of a gantt diagram for the overall project management.
\end{abstract}

\section{Acknowledgement}

The authors would like to acknowledge the support by the DFG Deutsche Forschungsgemeinschaft for the Research Training Group 2193 Adaptation Intelligence of Factories in a Dynamic and Complex Environment.

\section{Introduction}

With the arising global competition amongst manufacturers and the overall increasing speed of technological development on the side of both consumer goods and production technology, traditional production companies have to face new challenges such as increased product and production complexity, reduced product life cycles and at the same time increasing market volatility [1-3]. Therefore, existing factories cannot maintain the same status and production system as it was usual in the past. In order to remain competitive in this highly dynamic and complex environment, production companies have to adapt to these challenges and change their production system in a very agile and flexible manner. They have to be able to change their production structure rapidly and reconfigure the existing system to react efficiently to the changing demand of new products [4].

Despite using new production technologies such as autonomous controlled AGVs or $3 \mathrm{~d}$ printing for rapid prototyping or other solutions that become highly anticipated in the 4th industrial revolution, it is necessary to change the way companies plan their reconfiguration of the existing system in order to exploit crucial time benefits as a first step [5]. Time and costs are key factors in the reconfiguration process. Considering the increasing required time for such complex planning projects it becomes evident that this time is not available in this fast changing environment. In addition to that, the costs for a reconfiguration project that needs to be planned and realized are also increasing because of the more and more specialized participants of the planning project [6]. This increasing interdisciplinary intensifies the complexity of a project which affects the planning costs additionally [7]. Taking these two contrary factors, more time and money needed but not available, into account, it spans a widely known dilemma of planning and especially of factory planning projects [8-10].

To manage the factory planning process a variety of several approaches, which are focusing on different specific topics such as the overall project management, or the maturity level of planning information, were developed $[11,12]$. Some approaches consider the whole planning process from the initiation by the corporate management to the realization of the physical aspects with a ramp-up phase of the production [13]. Most of the approaches 
do not consider a synchronization of the important part of planning the production system and the equal important planning of the building that is necessary for the factory. Such synchronizing that considers parts of information that is important for both aspects can reduce time and costs for the whole planning project. Taking also into account that a more agile and modular approach of planning is combined with this synchronization can reduce the overall complexity of planning projects and opens up huge potential for dealing with the introduced struggles of production companies in questions of reconfiguration and adaptation.

This paper deals with the combination of a modular factory and building planning approach that will use component based synthesis to partly automatically align the necessary planning tasks along an overall planning workflow. This will allow a better project management of the whole factory planning project.

\section{Modular Factory Planning}

When it comes to the actual factory planning process the planning team can make use of several approaches that will give them a useful guideline for the step by step planning of factory planning projects. Classical approaches focus on separated planning tasks, which are packed into specific phases. These phases are aligned into a sequential order and are then processed step by step [14-16]. The results of one phase form the basis for the following phases. These established approaches are very stiff because of their phaseoriented sequential order and cannot be arranged to a specific planning project accordingly as their definition does not include such dynamic adaptations (see Figure 1).

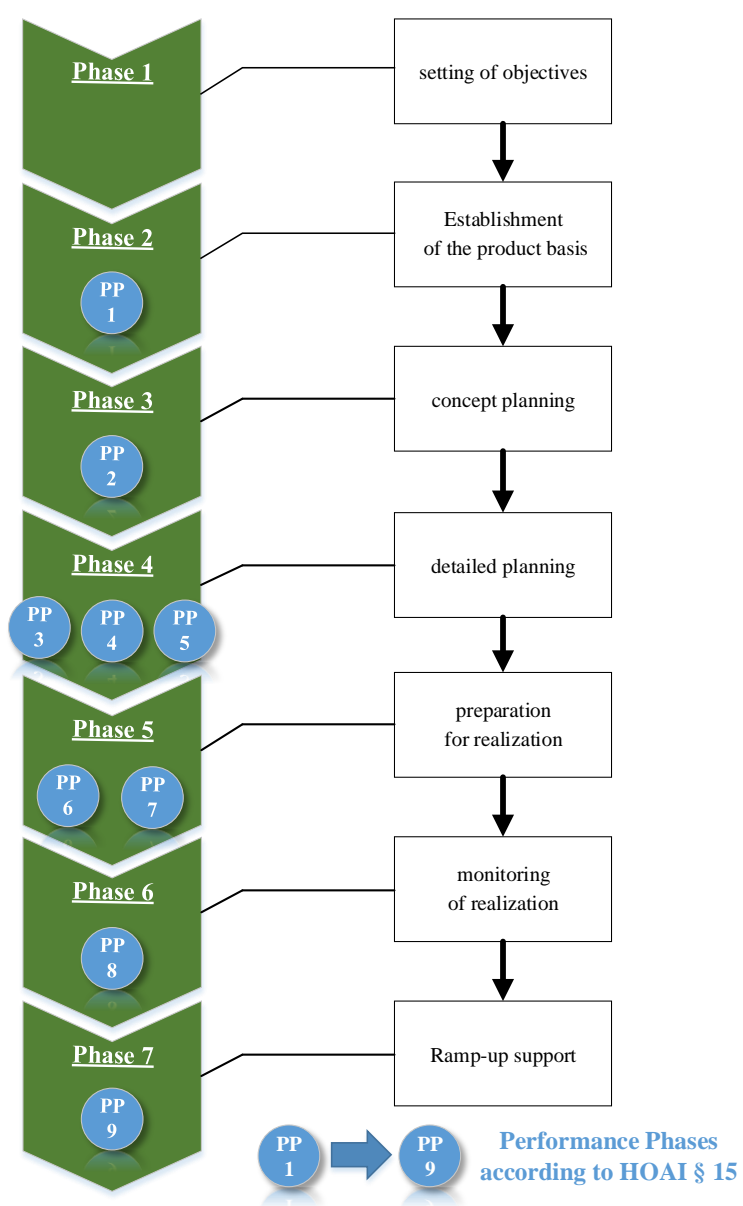

Figure 1: Factory planning model according to VDI 5200 [13]

As mentioned before the dynamics of changing elements that effect one company and therefore the accompanied production system are rising. These dynamics also affect the planning why it is necessary that given planning models are as flexible as possible. Not only by arranging the necessary steps tailored to the individual planning project but also the possibility to adapt the worked out planning workflow to new information and circumstances.

In order to deal with these challenges, new approaches were developed that focus on flexibility in planning and be adaptable to new conditions. Instead of forming huge planning phases that consists of a lot of planning tasks along the factory planning workflow, specific tasks and their information where formed into planning modules $[8,12,17]$. These modules consists of defined in- and outputs, which also determine the interdependencies of several other modules (see Figure 2). 


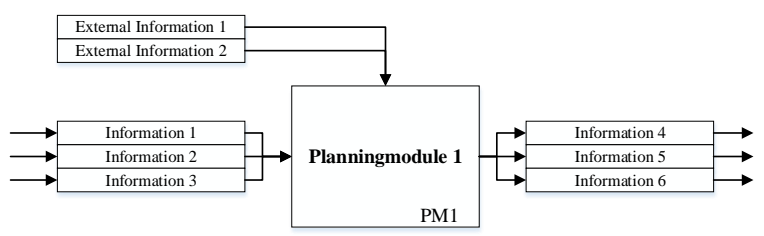

Figure 2: Exemplary setup of a planning module [17]

With the help of such modular approaches, it is possible to arrange the planning workflow according to the specific and individual planning project as a first step. The arrangement of the several tasks is not fixed anymore as it is given in classical, sequential approaches (see Figure 3). As a second step, it is possible to adapt the planning workflow based on new information by interchanging affected planning modules with less effort. An overall benefit of the modular approach is the reduction of complexity that arise with the accompanied dynamics. By splitting a bigger task or planning phase into smaller, more manageable planning modules, the complexity becomes less overwhelming $[11,17]$.

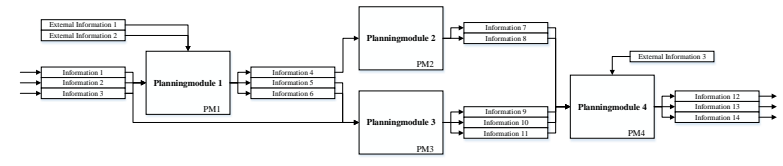

Figure 3: Example of a planning workflow

The modular planning approaches that are developed for the area of factory planning do not consider the actual support of generating the needed information. The action that takes places inside each module is seen as a black box and therefore no guidance of creating outputs is given. Therefore, it is necessary to combine the linked information of each module with specific methods that can be used to generate the required information (see Figure 4). In addition to this, more aspects added to the setup of a planning module such as the needed resources such as time and budget in combination with the identified personnel can enrich and support the process of generating planning information. This makes it even easier for the planning team to coordinate the participants who can be very interdisciplinary. The interdisciplinary teams need a higher level of transparency, which is not given with only in- and output information.

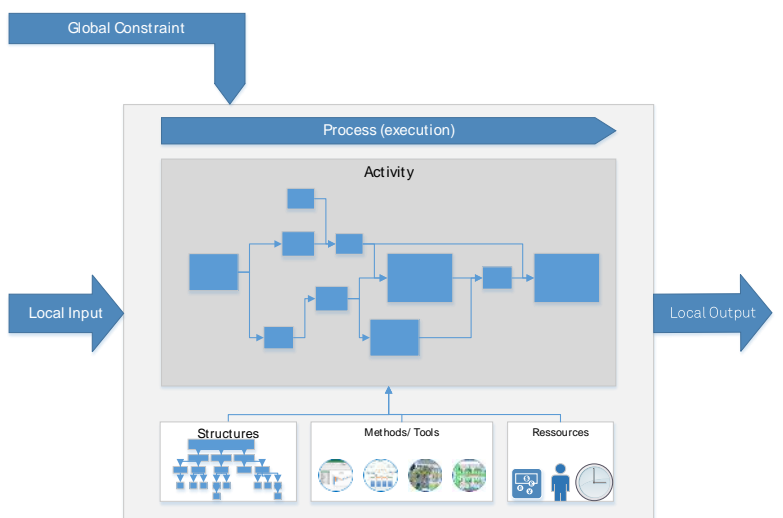

Figure 4 : Enhanced setup of a factory planning module

Especially when it comes to the combination of the expertise of production planning and building construction it becomes evident that a higher level of transparency is necessary in order to be able to coordinate the interdisciplinary participants efficiently. As an example, a small planning workflow is shown in Figure 5.

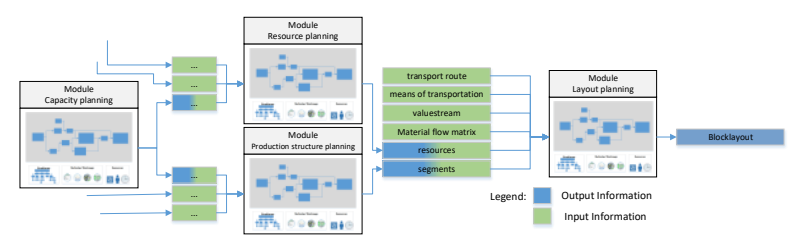

Figure 5: Example of a planning workflow [18]

The interconnections between the used modules can be reach a high and very complicated number that needs to be dealed with in an efficient way. Therefore, the management of this information exchange between the different modules and the connected disciplines is crucial for efficient planning. An exchanging model as a database in which the generated information can be stored and later on used for a streamlined workflow of production and building planning is necessary. In addition to that a more specific focus on the interdisciplinary collaboration and usage of information that has to be generated throughout the planning project needs to be an important aspect for managing interdisciplinary planning projects like in the field of factory planning.

\section{Modular Building Planning}

In order to evaluate the technical and economic effects of adapting a production system, it is necessary to also consider the flexibility and adaptability of the factory building. 
If an adaptation of a production system becomes necessary, the overall evaluation must also take into account the building flexibility and any needed adaptations for example to the load-bearing structure or similar. For this purpose, it is essential to determine the current condition of the factory building. This is conventionally only achievable by time-consuming examination of the as-built documentation, which consists of a large number of documents with highly inhomogeneous file formats. On the one hand, incorrect data within the documentation can lead to wrong decisions. On the other hand, the actuality of the as-built documents is a problem because adjustments after completion of the factory building are not always noted or implemented in the as-built documents. For this reason, inspections and also laser scanning technologies are used to record the current status of the factory building. [18]

Conventional planning methods within the construction industry are not able to support necessary adaptations of the building structure as well as in the area of factory systems in an interdisciplinary and efficient way. In this context, the approach of the Building Information Modeling method, in which a BIM model that contains all the necessary information in one database is used, is promising. Building Information Modeling is a method in the construction industry that includes the generation and the management of digital, virtual representations of the physical and functional characteristics of a building. The digital building models represent an information database around the building, which can be used to optimize decision-making over the entire life cycle. [19]

The BIM method can be distinguished from conventional planning methods by linking 3D planning with non-geometric information such as quality information. At the point wheregeometric information will be linked with non-geometric information, the building model (3D) develops into a building information model $(3 \mathrm{D}+\mathrm{i})$.

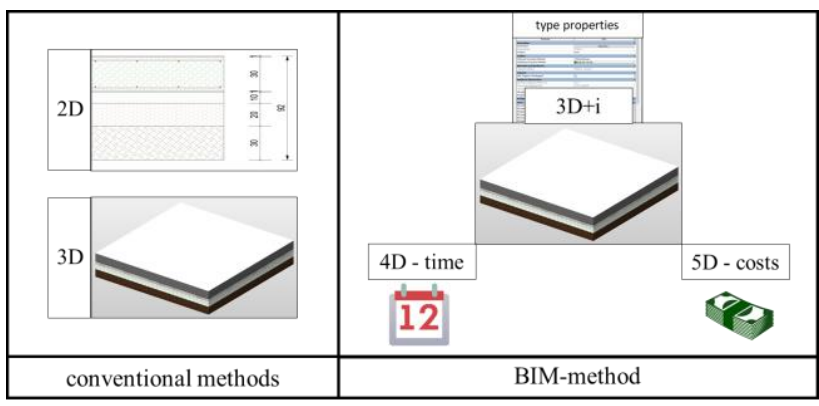

Figure 6: Separation of conventional design and construction from design and construction with BIM [20]

Scheduled aspects are linked to the components or objects e.g. time estimates or effort values and considered as the fourth dimension. For this purpose, the BIM model can be linked with the corresponding time schedule as figure 6 indicates.. [20]

In order to consider the costs, the price-forming and cost-relevant information is combined with the objects of the BIM model, which is done in the fifth dimension. By consolidating the objects with individual items of a bill of quantities, a model-based cost calculation can be automatically carried out. [20]

Furthermore, the use of the BIM method offers the potential to optimize processes under consideration of the whole life cycle of a factory, such as supporting the factory adaptation process and its more efficient and faster implementation [5]

With the purpose of make interdisciplinary planning more efficient, while taking factory planning and building planning into account, it is advisable to implement the modular planning approaches of the factory within building planning and combine them with the BIM method. Therefore, it is recommended to consider the components respectively objects of the building as modules. A module can also contain various submodules, which are regarding to different parameters with associated characteristics or values. For example, the component floor slab can function as a (main) module and the influencing components such as underground, base layer, cleanliness layer, concrete floor slab and coating system can be considered as submodules, which are then provided with the necessary parameters and associated characteristics.

\section{Beneficial hybrid Approach}

With aim of being able to use the modular planning approach in combined form for factory planning and 
building planning, it is first necessary to develop planning modules for both disciplines. In this context it is quite possible that individual planning modules affect both disciplines and that relevant information from these must be taken into account.

For this purpose, the developed planning modules include submodules which contain information in a higher level of detail, which is declared as parameters / attributes. The associated values can take on different characteristics that reflect the information at the lowest level. Essential is the respective database, which describes the degree of information of the different modules. For a realistic evaluation of factory adaptation processes, the interdisciplinary data must be stored and linked in the modules for each discipline. For this reason, all data required for a possible factory adaptation process is transferred to the BIM model, which also functions as a knowledge database. The planners responsible for the various planning modules are able to find all the information within the BIM model and use it for evaluations.

Through the hybrid approach of modular planning and the use of satisfiability module theories on an interdisciplinary level, different planning data can be correlated and evaluated. Based on the described approach, it is possible to efficiently and quickly evaluate the flexibility of a factory building with regard to a factory adaptation process in a partly automated way. The hybrid approach of modular planning also offers the advantage that the respective modules can be linked together clearly at a global level. As soon as more detailed information is required, the associated submodules must be viewed or created with the linked parameters and their characteristics, so that links can be made on a more detailed, deeper level. In this way, it is possible to determine the resulting impacts and restrictions partly automated and do not have to be carried out by manual, repetitive activities.

In the course of processing the approach, various planning modules for a factory adaptation process were defined, which are subdivided into the areas "communal planning", "product system planning" and "building planning". Within the three areas, the related modules are arranged as shown in Figure 7.

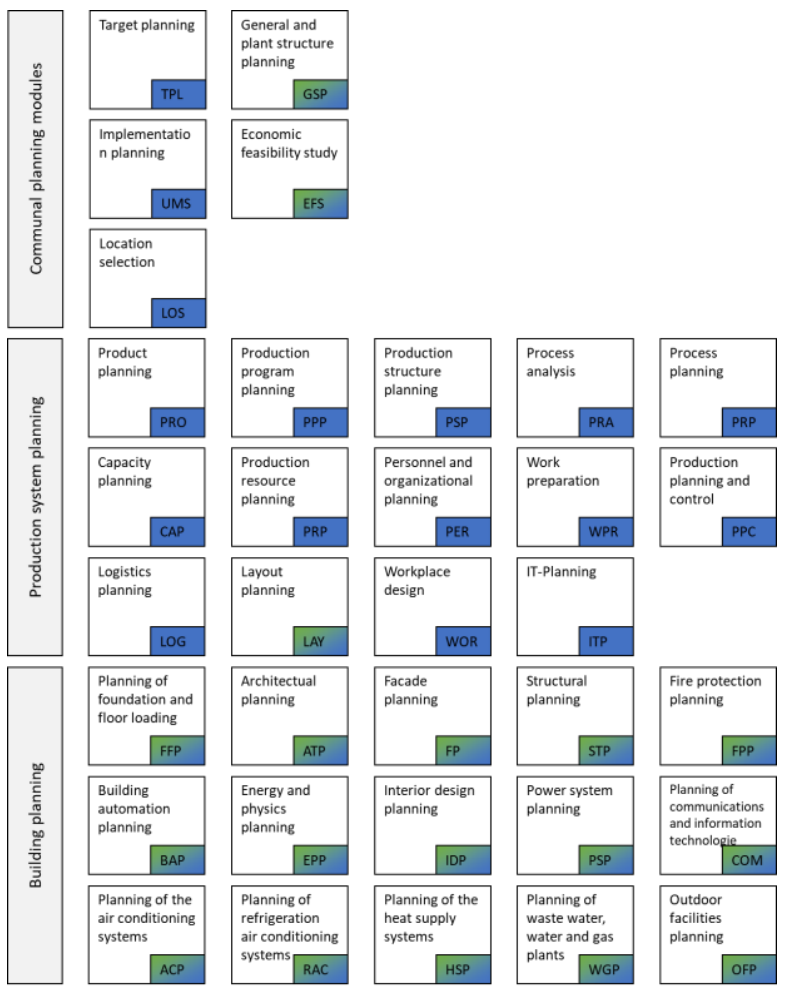

Figure 7: Defined planning modules under consideration of the different disciplines [11]

\section{Type Based Synthesis and SMT- Solving as technical solution}

Software synthesis allows the construction of a program from a logical specification rather than writing program code from scratch [21]. It is used to generate optimal code sequences or to automate simple programming tasks [22]. However, the synthesis is not limited to software in programming languages, but also allows the composition of system configurations or BPMN 2.0 processes [23]. Particularly advantageous is the synthesis of programs in complex systems such as systems with concurrent processes [21].

In the software synthesis different approaches of the calculation as well as the methods of the specification can be distinguished. Some works can be characterized by the use of temporal logic and automata theory, while others can be characterized by the use of deductive methods and type theory [23].

This paper presents a component-based synthesis framework, which can be assigned to functional synthesis with a semantic search space. The Combinatory Logic Synthesizer Framework, or (CL)S 
for short, is a type-based framework for synthesizing software from a set of components specified in a library. The framework was developed in the programming language Scala and is used in this paper. In addition to the synthesis, the framework also enables the immediate execution of the synthesis result. The implementation in the programming language Scala allows the synthesis results to access existing Java and Scala libraries. The Framework (CL)S was developed at the Chair for Software Engineering of the Faculty of Computer Science of the TU Dortmund University [23].

(CL)S uses a structured collection of components and a target type for the synthesis. In the context of (CL)S, the components are called combiners. In order to make components usable for synthesis, they must be specified according to the specifications of the framework.

The individual components are classified by two data types: The native type (e.g. integer or string) and the semantic type, which describes the content of the respective module. The semantic types also enable the transfer of annotations and techniques from the Semantic Web into the type system.

Each component also has input parameters that are typed in the same way. In synthesis, a pair of natives and semantic data type is specified as the synthesis target, and an attempt is made to create a compilation of components that meets the target and simultaneously covers all input parameters with other components. We use the framework to automatically merge modules from factory design and civil engineering into complete solutions by specifying a planning goal. Chapter 6 shows how this is done using an example.

In many areas of computer science important problems can be reduced to formulas in a certain logic and their fulfillability. Some of these problems can, for example, be formulated in propositional logic and checked for their fulfillability with modern SAT solvers [24]. Other problems, on the other hand, can be formulated more naturally and compactly in logics such as predicate logic, since this logic is more expressive than propositional logic through the use of non-Boolean variables, functions, predicates and quantifiers. The expressiveness of the formulas can be further enhanced by the use of theories such as arithmetic, arrays or quantifiers. So-called satisfiability modulo theories, SMT for short, can determine the satisfiability of these formulas [25].
Due to the progress in technology and research of the Satisfiability Modulo Theories, there is a multitude of powerful and highly developed SMT solvers such as Alt-Ergo, Beaver, Boolector, CVC4, MathSAT5, openSMT, SMTInterpol, Sonolar, STP, veriT, Yices and Z3 [24]. The progress in the development of SMT solvers is attributed to the initiation of the annual SMT solver competition SMT-COMP and the introduction of the standardized input and output format for benchmarks called SMT-LIB.

The use of this technique is necessary in this context, which (CL)S does not take into account in the construction of solutions numerical constraints. Consequently, an SMT solver is used to ensure that a generated solution can be completed within a certain time window, for example.

The entire workflow and the interaction between (CL)S and the SMT Solver are presented in a use case in Chapter 6.

\section{Use Case}

In order to be able to recognize and evaluate the possibilities and advantages of the hybrid, modular planning approach for factory adaptations, a use case was developed. In this use case, the effects of the integration of a production robot are considered. For the use case, a database is first created for the planning modules examined, so that a symbiosis of the data from the various disciplines can subsequently be achieved. For a partly automated evaluation using the hybrid approach, all data from factory planning is transferred to the BIM model.

The database consists of (see Figure 8: Database for the use-case-specific BIM Model):

- Data of the production machine to be implemented

- Data of the building components within the factory, which may be affected by the adaptation and influence qualities, costs and deadlines.

- Data of the defined planning modules

- Interfaces and dependencies of the various planning modules and their submodules and parameters 


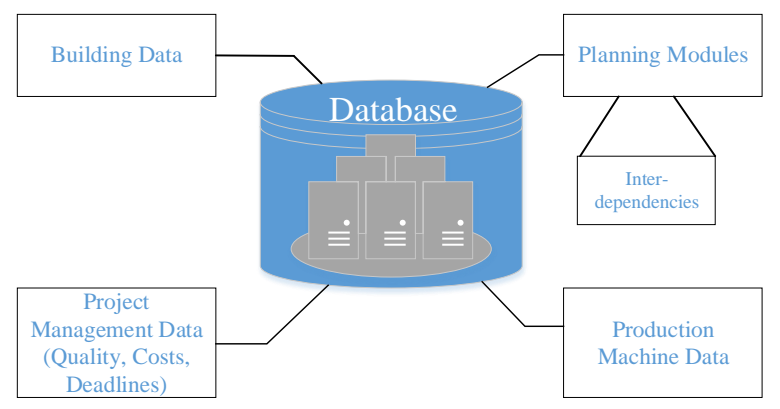

Figure 8: Database for the use-case-specific BIM Model

When using a new production machine such as a robot, the layout planning is directly affected regarding to factory planning and building planning. For this use case, the layout planning module was examined in more detail and the data from the various specialist disciplines compiled in a symbiosis. By considering the data within the BIM model, it is possible to identify geometric restrictions and check the technical feasibility. Another advantage is that the modules can be linked together and partly automated examinations can be carried out using the parameter values stored in the submodules, so that, for example, requirements from the planning modules of factory planning can be compared and evaluated with the existing specifications of the planning modules of building planning. In the present use case, for example, the requirement for a compressed air connection is made from the factory planning. This requirement allows the stored machines to be evaluated in the "production machines" module. With the help of the CLS, it can be selected partly automatically which machines are usable for the present use case. Furthermore, the "floor slab" module from building planning can be used to partly automatically determine which loads can be taken up by the floor slab. Finally, the previously selected robots are checked again by the CLS and compared with the specifications of the floor slab module. This procedure allows all dependencies to be displayed and partly automatically verified, so that finally only possible solutions are output.

The composition of the individual modules takes place in CLS by generating sequences of function calls. Each module is represented by a function with input parameters and an output type. For example, the module "Production Machines" is represented by the function "(Compressed Air Required for Painting) -> "Production Machine (alpha)", where alpha can stand for any machine of the type "Production Machine" (see Figure 9). When using the module, the CLS searches for modules that fulfill the input parameters of the function by their respective output type. This results in concatenations of function calls, which in turn represent the sequence of modules used. It is important to mention that all possible variations of processes are generated, which are correct based on their typing. In the second run, the SMT solver then sorts out the solutions that do not correspond to the numerical constraints (such as the load limit). For more details see [18].

The preceding synthesis has resulted in a series of variants of possible workflows that meet the specified planning objective and are composed of work steps and processes (modules) known to the planner. In addition, however, a check is necessary to ensure that the solution variants, which are to be made available to the planner again at the end, also comply with project-specific numerical constraints (budget limits, time limits, resource restrictions) and module-specific constraints (e.g. mutual exclusion of certain modules). These checks are carried out by an SMT solver.

In our case this has been realized exemplarily by means of Scala Graph, but it is also possible to use other SMT-Lib compatible solvers like Z3.

For the enforcement of the global constraints, such as the maximum duration or the maximum budget, simple functions can be set up. For local constraints the RefuseNode function of Scala-Graph is used. It creates a kind of "blacklist" of nodes that must not be used in conjunction with the node in question. RefuseNode functions can be regularly implemented in the CLS Combinator. 


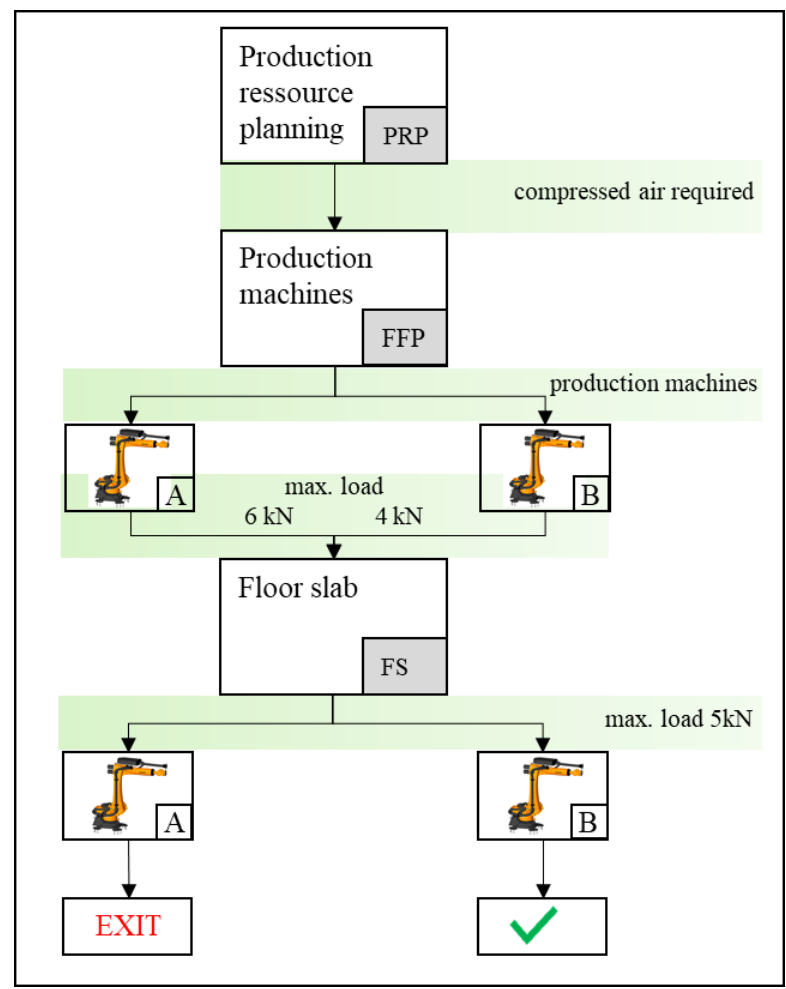

Figure 9: Workflow of using hybrid approach by using modular planning and symbiosis of the interdisciplinary data

\section{Conclusion \& Outlook}

The developed modular factory planning approach in this paper has shown a new way of synchronizing the mainly separately done planning of the production system and the building. The synchronization which considers parts of information that is important for both aspects can reduce time and costs for the whole planning project. The information data between different planning modules can be exchanged in a transparent and lossless way using a BIM model. In combination with a component based synthesis the necessary planning tasks can be aligned partly automated along an overall planning workflow. This reduces the overall complexity of planning projects and offers a huge potential for dealing with the challenge of production companies regarding reconfiguration and adaption of the existing production system.

Overall, the hybrid approach of modular planning offers a promising improvement of the collaboration between production system planning and building planning. The requirements and the information of the individual planning modules among each other can be analysed by the component based synthesis. Thus it is possible to partly automated examine, if all requirements between the modules were met by potential planning solutions. That automated support for planners makes future factory planning projects more manageable and can help to avoid planning errors in order to reduce costs and time. The approach of merging interdisciplinary data within a BIM-model, the associated creation of modules and the composition of these modules into complete models in compliance with the project constraints through the use of technologies such as synthesis and SMT solving is also applicable to other domains as well as airport buildings or health centers.

\section{References}

[1] D. Mourtzis, M. Doukas 2014, Design and Planning of Manufacturing Networks for Mass Customisation and Personalisation: Challenges and Outlook, Procedia CIRP, 19, 1 - 13. DOI: 10.1016/j.procir.2014.05.004.

[2] H. ElMaraghy, G. Schuh, W. ElMaraghy, F. Piller, P. Schönsleben, M. Tseng, A. Bernard 2013, Product variety management, CIRP Annals, 62 (2), 629 - 652. DOI: 10.1016/j.cirp.2013.05.007.

[3] D. Mourtzis, M. Doukas 2014, The Evolution of Manufacturing Systems, in Design and management of lean production systems, Vol. 75 (Eds: P. Semanco, V. Modrák), Business Science Reference, an imprint of IGI Global. Hershey PA.

[4] S. Weyer, M. Schmitt, M. Ohmer, D. Gorecky 2015, Towards Industry 4.0 - Standardization as the crucial challenge for highly modular, multi-vendor production systems, IFAC-PapersOnLine, 48 (3), 579 - 584. DOI: 10.1016/j.ifacol.2015.06.143.

[5] T. Delbrügger, F. Döbbeler, J. Graefenstein, H. Lager, L. T. Lenz, M. Meißner, D. Müller, P. Regelmann, D. Scholz, C. Schumacher, J. Winkels, A. Wirtz, F. Zeidler 2017, Anpassungsintelligenz von Fabriken im dynamischen und komplexen Umfeld, $Z W F, 112$ (6), 364 - 368. DOI: $10.3139 / 104.111731$.

[6] A. Kampker, A. Meckelnborg, P. Burggräf, T. Netz 2013, Factory Planning Scrum: Integrative Factory Planning with Agile Project Management, in International Conference on Competitive Manufacturing: COMA '13 30 January- 01 February 2013, Stellenbosch, South Africa proceedings (Eds: D. Dimitrov, C. Schutte). Stellenbosch, South Africa.

[7] A. Kampker, A. Meckelnborg, P. Burggräf, T. Welter 2012, Integrative Factory Design by Efficient Interaction Models, in POMS 23rd Annual Conference-Proceedings of the 23rd Annual Conference of the Production and Operations Management Society "Socially Responsible 
Operations", Chicago, Illinois, U.S.A. April 20 to April 23, 2012 (Eds: T. Schoenherr). Chicago.

[8] H. W. J. Rittel, M. M. Webber 1973, Dilemmas in a general theory of planning, Policy sciences integrating knowledge and practice to advance human dignity the journal of the Society of Policy Scientists, 4 (2), 155 - 169.

[9] R. Hilchner 2012, Typenorientiertes LösungsraumManagement in der Fabrikplanung, 1st ed., Edition Wissenschaft Apprimus, Vol. 2012,13, ApprimusVerl., Aachen.

[10] A. Kampker, P. Burggräf, M. Krunke, K. Kreisköther, H. Voet, M. Backs 2014, Das Aachener Fabrikplanungsvorgehen, $w t$ Werkstatttechnik online, 104 (4), 192 - 196.

[11] A. Meckelnborg 2015, Integrative Fabrikplanung durch effiziente Koordinationsmodelle, 1 st ed., Ergebnisse aus der Produktionstechnik Produktionssystematik, Vol. 2015,23, ApprimusVerl., Aachen.

[12] M. Krunke, Reifegradmanagement in der Fabrikplanung 2017, Dissertation, RWTH Aachen.

[13] Verein deutscher Ingenieure e.V., Fabrikplanung Planungsvorgehen, Beuth, Berlin 2011, 03.100.99 (5200).

[14] B. Aggteleky 1987, Fabrikplanung: Werksentwicklung und Betriebsrationalisierung, 2nd ed., Hanser, München.

[15] H. Kettner, J. Schmidt, H.-R. Greim 1984, Leitfaden der systematischen Fabrikplanung, Hanser, München, Wien.

[16] C.-G. Grundig 2015, Fabrikplanung: Planungssystematik - Methoden - Anwendungen, 5th ed., Hanser, München.

[17] J. C. Nöcker 2012, Zustandsbasierte Fabrikplanung, 1st ed., Edition Wissenschaft Apprimus, Vol. 2012,6, Apprimus-Verl., Aachen.

[18] L. T. Lenz, J. Graefenstein, J. Winkels, M. Gralla 2019, Smart Factory Adaptation Planning by means of BIM in Combination of Constraint Solving Techniques, Dortmund.

[19] M. Egger, K. Hausknecht, T. Liebich, J. Przybylo 2013, BIM-Leitfaden für Deutschland,:

Forschungsprogramm ZukunftBAU, Endbericht, Bundesinstitut für Bau-, Stadt- und Raumforschung (BBSR) und Bundesamt für Bauwesen und Raumentwicklung (BBR), Berlin.

[20] M. Gralla, L. T. Lenz 2017, Digitalisierung im Baubetrieb, in Festschrift zum 60. Geburtstag von Univ.-Prof. Dr.-Ing. Christoph Motzko (Eds: J. Fenner).

[21] R. Bodik, B. Jobstmann 2013, Algorithmic program synthesis: introduction, International Journal on Software Tools for Technology Transfer, 15 (5-6), 397 - 411. DOI: 10.1007/s10009-013-0287-9.

[22] S. Jha, S. Gulwani, S. A. Seshia, A. Tiwari 2010, Oracle-guided component-based program synthesis, in ACM/IEEE 32nd International Conference on Software Engineering, 2010 (Eds: J. Kramer, J. Bishop, P. Devanbu, S. Uchitel), IEEE. Piscataway, NJ.
[23] J. Bessai, A. Dudenhefner, B. Düdder, M. Martens, J. Rehof 2014, Combinatory Logic Synthesizer, in Leveraging applications of formal methods, verification and validation, Vol. 8802, LNCS sublibrary. SL 1, Theoretical computer science and general issues (Eds: T. Margaria-Steffen, B. Steffen), Springer. Heidelberg.

[24] C. Barrett, C. Tinelli 2018, Satisfiability modulo theories, in Handbook of Model Checking, Springer.

[25] L. de Moura, N. Bjørner 2008, Z3: An Efficient SMT Solver, in Tools and Algorithms for the Construction and Analysis of Systems, Vol. 4963, Lecture Notes in Computer Science (Eds: C. R. Ramakrishnan, J. Rehof), Springer-Verlag Berlin Heidelberg. Berlin, Heidelberg. 